\title{
An Excess of Low-mass X-Ray Binaries in the Outer Halo of NGC 4472
}

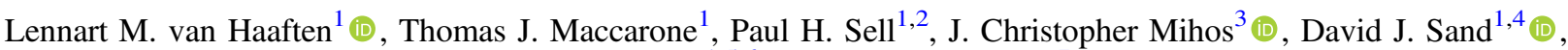 \\ Arunav Kundu ${ }^{1,5,6}$, and Stephen E. Zepf ${ }^{7}$ \\ ${ }^{1}$ Department of Physics and Astronomy, Texas Tech University, Box 41051, Lubbock, TX 79409-1051, USA; L.vanHaaften@ttu.edu \\ ${ }^{2}$ Physics Department, University of Crete, 71003 Heraklion, Crete, Greece \\ ${ }^{3}$ Department of Astronomy, Case Western Reserve University, 10900 Euclid Avenue, Cleveland, OH 44106, USA \\ ${ }^{4}$ Steward Observatory, The University of Arizona, 933 N. Cherry Avenue, Tucson, AZ 85721, USA \\ ${ }^{5}$ Eureka Scientific, Inc., 2452 Delmer Street, Suite 100, Oakland, CA 94602, USA \\ ${ }^{6}$ Computational Physics, Inc., 8001 Braddock Road, Suite 210, Springfield, VA 22151, USA \\ ${ }^{7}$ Department of Physics and Astronomy, Michigan State University, East Lansing, MI 48824, USA \\ Received 2017 July 18; revised 2017 October 24; accepted 2017 November 14; published 2018 January 17
}

\begin{abstract}
We present new Chandra observations of the outer halo of the giant elliptical galaxy NGC 4472 (M49) in the Virgo Cluster. The data extend to $130 \mathrm{kpc}\left(28^{\prime}\right)$, and have a combined exposure time of $150 \mathrm{ks}$. After identifying optical counterparts using the Next Generation Virgo Cluster Survey to remove background active galactic nuclei and globular cluster (GC) sources, and correcting for completeness, we find that the number of field low-mass $\mathrm{X}$-ray binaries (LMXBs) per unit stellar $V$-band light increases significantly with the galactocentric radius. Because the flux limit of the complete sample corresponds to the Eddington limit for neutron stars in NGC 4472, many of the $\sim 90$ field LMXBs in this sample could host black holes. The excess of field LMXBs at large galactocentric radii may be partially caused by natal kicks on black holes and neutron stars in binary systems in the inner part of the galaxy. Furthermore, because the metallicity in the halo of NGC 4472 strongly decreases toward larger galactocentric radii, the number of field LMXBs per unit stellar mass is anticorrelated with metallicity, opposite to what is observed in GCs. Another way to explain the spatial distribution of field LMXBs is therefore a reversed metallicity effect, although we have not identified a mechanism to explain this in terms of stellar and binary evolution.
\end{abstract}

Key words: galaxies: abundances - galaxies: halos - galaxies: individual (NGC 4472) - stars: evolution $\mathrm{X}$-rays: binaries

\section{Introduction}

X-ray observations of old stellar populations such as those of elliptical galaxies, spiral galaxy bulges, and globular clusters (GCs) reveal numerous, bright X-ray point sources. These are almost exclusively low-mass X-ray binaries (LMXBs; Trinchieri \& Fabbiano 1985), stellar systems in which a neutron star (NS) or black hole $(\mathrm{BH})$ accretes matter from a low-mass (mass $\lesssim 1.5 M_{\odot}$ ) companion star overfilling its Roche lobe. Their population sizes and properties are important for our understanding of stellar and binary evolution (e.g., Tauris \& van den Heuvel 2006).

The formation rate of LMXBs depends on many factors, the two most important being the total stellar mass and the number density of stars, both of which are clearly demonstrated by observations. The number of LMXBs is positively correlated with the total stellar mass, as low-mass stars contain the majority of the stellar mass in a galaxy (e.g., Gilfanov 2004). The number of LMXBs is also positively correlated with the stellar density, even per unit stellar mass. Many LMXBs are found in GCs, which contain about two orders of magnitude more LMXBs per unit mass than in the field (Katz 1975). In early-type galaxies, roughly half of LMXBs reside in GCs (e.g., Sarazin et al. 2000; Angelini et al. 2001; Kundu et al. 2002). This can be explained by the high frequency of dynamical interactions in these stellar systems (e.g., Clark 1975).

These and other factors affect the spatial distribution of LMXBs in a galaxy. While the distribution of LMXBs is strongly affected by the distributions of the stellar mass and
GCs, varying metallicity and migration caused by supernova kicks could alter it as well.

\subsection{Kicks Received by Black Holes and Neutron Stars}

Natal kicks of NSs generally have velocities of several hundreds of $\mathrm{km} \mathrm{s}^{-1}$ (Lyne \& Lorimer 1994). There is also evidence that $\mathrm{BHs}$ receive high natal kick velocities, close to those of NSs (e.g., Brandt et al. 1995; Fragos et al. 2009; Repetto \& Nelemans 2015), as well as evidence for misaligned spins caused by asymmetric kicks (e.g., Hjellming \& Rupen 1995; Ingram et al. 2009).

However, some NSs (Pfahl et al. 2002; Podsiadlowski et al. 2004; van den Heuvel 2007) and BHs (e.g., Miller-Jones et al. 2009; Reid et al. 2014) are known to receive weak kicks. Overall, the BH kick velocity distribution is not as well constrained as that of NSs and current evidence points to a fairly wide range of $\mathrm{BH}$ kick velocities.

\subsection{The Metallicity Effect for LMXBs}

The metallicity effect is the observation that metal-rich GCs have a higher chance to host an LMXB than metal-poor clusters. High metallicity is indicated by late collective spectral type and low galactocentric distance.

Kundu et al. (2002) established the metallicity effect using Chandra X-ray Observatory data of NGC 4472, finding that metal-rich (red) GCs are about 2.7 times more likely to host an LMXB than metal-poor (blue) clusters, and also showed clearly that the effect was not just due to a possible dependence of 
color on galactocentric radius or cluster luminosity (i.e., stellar population size, see also Sarazin et al. 2003).

\subsubsection{Proposed Explanations for the Metallicity Effect}

If metal-rich environments would produce increased numbers of massive stars, or stars with large radii, more X-ray binaries would be expected to form, possibly explaining the metallicity effect.

Grindlay (1987, 1993) argued that a flatter initial mass function implies a higher number of NSs, hence more LMXBs, possibly (or partially) explaining the metallicity effect.

Bellazzini et al. (1995) argued for a dependence on metallicity of stellar radii as well as stellar masses, which would increase tidal capture rates and fill Roche lobes at larger orbital separations. Ivanova et al. (2012) argued that in metalrich GCs, red giants are more massive and more numerous than in metal-poor ones. This leads to higher collision and binary exchange frequencies.

Other explanations involve the timescale of binary evolution. Iben et al. (1997) and Maccarone et al. (2004) pointed out that metal-poor donors have less line cooling and therefore stronger irradiation-induced stellar winds, and that as a result, these binaries lose more angular momentum, evolve faster, and live shorter.

Another resolution was proposed by Ivanova (2006), who found that metal-rich donor stars in the mass range $0.85-1.25 M_{\odot}$ have an outer convection zone, and as a result they can experience magnetic braking, which is an important source of angular momentum loss that drives binary evolution. Stars with masses in the upper part of this range may no longer exist in old populations, but their descendants can still be X-ray binaries with lower donor masses.

\subsection{Aim of this Work}

Some of the models for the metallicity effect described in the previous section, such as increased stellar winds and outer convection zones, have the same effect regardless of stellar interaction rates, and therefore predict the same metallicity effect in GCs and the field. The remaining mechanisms work in dynamical environments for additional reasons than in lowdensity environments, and predict a stronger metallicity effect in GCs compared with the field. More NSs, larger stellar radii, and higher stellar masses result in more captures in GCs, while they also result in, respectively, more primordial LMXBs, more easily filled Roche lobes, and stronger magnetic braking in environments of any stellar density.

To disentangle the cause of the metallicity effect, one can test whether the metallicity effect is the same or different in an environment where dynamical interactions between stars and binaries do not contribute to the formation of LMXBs, i.e., the field. If the metallicity effect is the same in the field, then increased stellar winds and outer convection zones may be the best explanation of the metallicity effect. If it is weaker in the field, then the other explanations are more likely. If the effect is stronger in the field, further explanations are needed, such as natal kicks.

Here, we present such a study of the metallicity effect in the field using new Chandra data of the outer halo of the giant elliptical galaxy NGC 4472 (M49), a member of the Virgo Cluster. The NGC 4472 halo is massive and extended, and Mihos et al. (2013) determined that the halo has steep color and metallicity gradients between 30 and $100 \mathrm{kpc}$, making it an excellent environment to test the metallicity effect. Our Chandra observations cover a larger angular radius and resolution than those obtained so far for any galaxy. We also used archival Chandra data of the inner region of NGC 4472 to complement the new data. We determined the number of field LMXBs per unit stellar light for a range of galactocentric radii, and hence a range of inferred metallicities.

\section{Observations and Data Analysis}

We adopted a distance of $16.3 \mathrm{Mpc}$ to NGC 4472 (Tonry et al. 2001), which is used to convert flux rates to luminosities. The center of the galaxy is located at R.A. $12^{\mathrm{h}} 29^{\mathrm{m}} 46^{\mathrm{s}} 80$, decl. $+08^{\circ} 00^{\prime} 01^{\prime \prime} 48$ (Chandra X-ray Center Data Archive).

\subsection{Chandra X-Ray Observations}

We obtained five new Advanced CCD Imaging Spectrometer I (ACIS-I) observations covering a large fraction of the halo of NGC 4472, with small overlaps between the fields (see Table 1 and Figure 1). The galactocentric radii of these fields range from $3 ! 2$ to $28.4(15-135 \mathrm{kpc}$, or $1-9$ effective radii, using $r_{\mathrm{e}}=3$ '.24 \pm 0 '.28 (Kormendy et al. 2009)). We also used an archival ACIS-S observation (ObsID 321) of the inner region, which is not covered by the new observations.

Sources in the Chandra observations were detected using the CIAO tool WAVDETECT (Freeman et al. 2002), with the SIGTHRESH parameter set at $10^{-6}$, and the SCALES values are a geometric progression with a constant factor of $\sqrt{2}$ between 2 and 16. Fluxes and their uncertainties were determined with the CIAO tool SRCFLUX with an absorbed power-law model with a photon index of 1.7 and a Galactic foreground neutral hydrogen column density based on the model by Dickey \& Lockman (1990). The average value in the direction of NGC 4472 is $N_{\mathrm{H}} \approx 1.6 \times 10^{20} \mathrm{~cm}^{-2}$, according to the HEASARC W3 $N_{\mathrm{H}}$ tool based on Dickey \& Lockman (1990), Kalberla et al. (2005).

We divided the galaxy into nine elliptical annuli on the sky with a constant width of $2 ! 63$ along the major axis, centered around the center of NGC 4472. The position angle of the major axis is $-31^{\circ}$ ( $31^{\circ}$ west of north), and constant ellipticity $1-b / a=0.28$, with $b / a$ the ratio between the lengths of the minor and major axes, following the shape of the isophotes used in Mihos et al. (2013) based on Kormendy et al. (2009), Janowiecki et al. (2010). For each X-ray source, we determined in which annulus it is located. A higher number of annuli would make Poisson errors on the number of X-ray sources per annulus larger than is practical.

Because star formation in NGC 4472 is expected to have ended at least several Gyr ago (Thomas et al. 2005; Baes et al. 2007), stars with masses over $1.5 M_{\odot}$ will no longer exist, so high-mass X-ray binaries are not expected in this field. This means that X-ray sources brighter than the sensitivity limit are either LMXBs or active galactic nuclei (AGNs). LMXBs in GCs and background AGNs are still included in Figures 1-3, but will be statistically removed per annulus using the method described in Section 2.2.3.

\subsubsection{Completeness Limits}

Further away from the aimpoint on each chip, the point spread function becomes larger and the sensitivity becomes worse. Figure 2 shows the fluxes of all sources against the off- 
Table 1

List of Chandra Observations of NGC 4472

\begin{tabular}{|c|c|c|c|c|c|c|}
\hline Observation ID & Instrument & Exposure Time (ks) & Data Mode & R.A. & Decl. & Start Date and Time \\
\hline 321 & ACIS-S3 & 39.59 & Very faint & 122946.90 & +080013.00 & 2000 Jun 12 01:47:47 \\
\hline 15756 & ACIS-I & 32.07 & Faint & 122904.40 & +074924.10 & 2014 Apr 16 09:50:28 \\
\hline 15757 & ACIS-I & 29.68 & Faint & 122845.00 & +080348.00 & 2014 Apr 18 00:01:11 \\
\hline 15758 & ACIS-I & 29.67 & Faint & 122943.20 & +081512.30 & 2014 Apr 20 13:52:10 \\
\hline 15759 & ACIS-I & 29.68 & Faint & 123043.80 & +080521.60 & 2014 Apr 25 18:45:47 \\
\hline 15760 & ACIS-I & 29.38 & Faint & 123014.20 & +075000.20 & 2014 Apr 26 03:26:34 \\
\hline
\end{tabular}

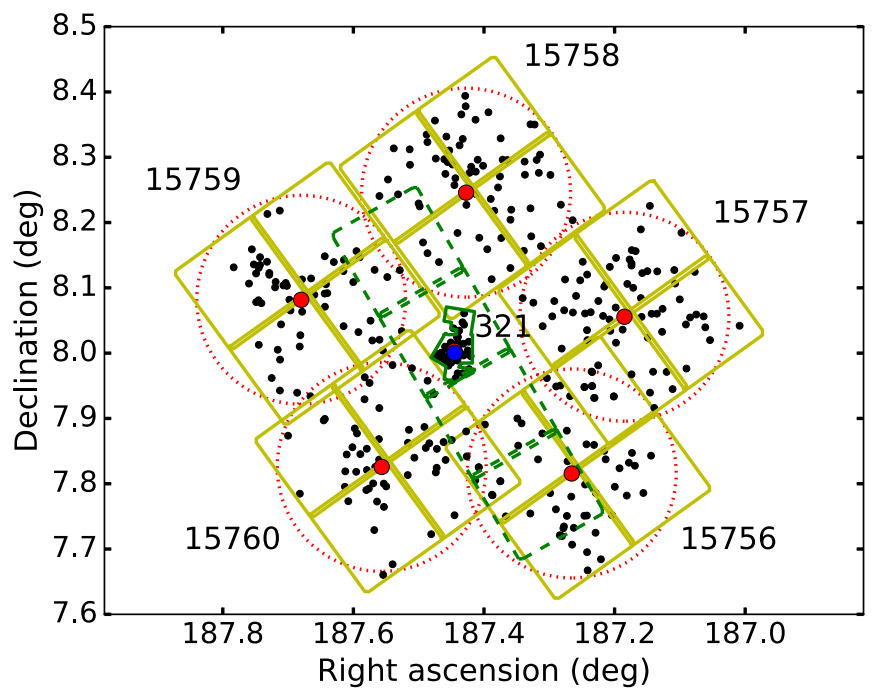

Figure 1. Chandra field of view of NGC 4472 used in this study. Yellow fields are the five new ACIS-I observations consisting of four CCDs each, with aimpoints shown as red circles. The archival ACIS-S field of the inner region is shown in dashed green, with the HST fields within the ACIS-S3 field in solid green. Chandra Observation IDs are shown in or next to the corresponding fields. The blue circle marks the center of the galaxy. The red dotted circles around each ACIS-I aimpoint depict the 9'.6 radial limits used to obtain a fluxlimited complete sample (Section 2.1.1). Black dots indicate all X-ray sources identified by WAVDETECT, including field and GC LMXBs, and background AGNs.

axis angle from the aimpoint of their respective observations (which is different from galactocentric radius). To have a fluxlimited complete sample, we discarded all sources fainter than a certain chosen flux limit, and also discarded sources located outside the off-axis angle corresponding to this flux limit (dashed blue line in Figure 2).

Choosing a low limiting flux means that more faint sources are included, but also that many sources far away from their respective aimpoints are discarded. A higher flux limit has the opposite effect, and in between is an optimum where over $50 \%$ of sources are retained. The fraction of all 344 sources identified by WAVDETECT that are included in a flux-limited complete sample (i.e., that is brighter than the flux limit and located within the corresponding off-axis radius) as a function of chosen off-axis angle cutoff is visualized in Figure 3. In this study, we adopted an angle of $9 ! 6$, at the maximum in the curve, corresponding to a completeness flux limit of $6.8 \times 10^{-15} \mathrm{erg} \mathrm{s}^{-1} \mathrm{~cm}^{-2}$ for the $0.5-7 \mathrm{keV}$ band $(\sim 16$ net counts for the faintest sources in the new halo observations). This converts to $2 \times 10^{38} \mathrm{erg} \mathrm{s}^{-1}$ for sources located at the distance of NGC 4472. At the end of Section 3, we present results for a smaller angle of 5!4 and a larger angle of $12^{\prime}$.

The aimpoints of the five ACIS-I fields are located at $12 ! 4$, $14 ! 8,14 ! 8,15 ! 4$, and $15 ! 8$ from the center of the galaxy. The

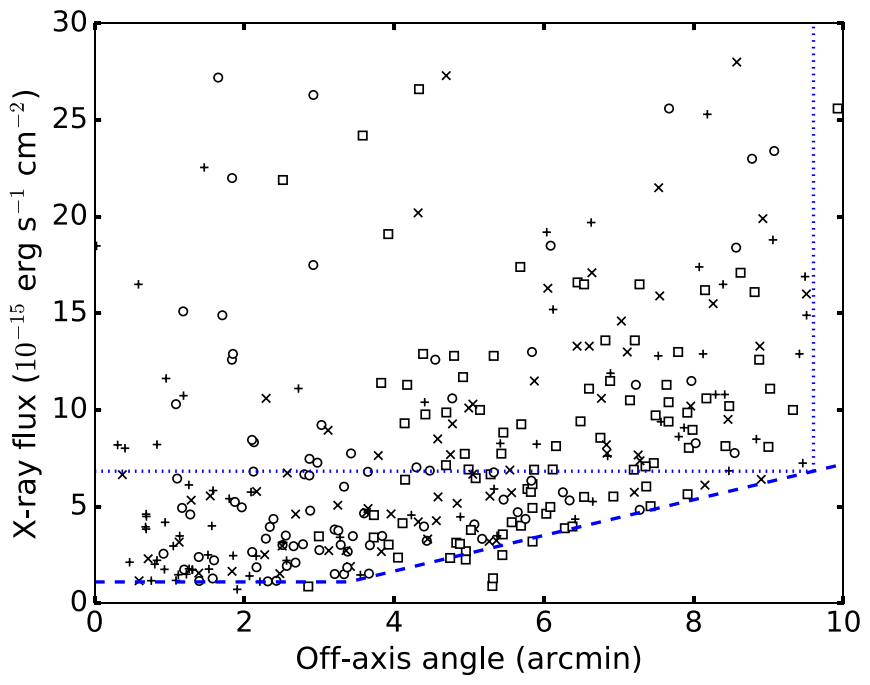

Figure 2. Full sample of $344 \mathrm{X}$-ray sources from the six observations listed in Table 1, with flux in the $0.5-7 \mathrm{keV}$ band plotted against angular distance from the respective aimpoints. Plus symbols indicate sources with galactocentric radii under $10^{\prime}$; crosses indicate those between $10^{\prime}$ and $14^{\prime}$; circles indicate those between $14^{\prime}$ and $18^{\prime}$; and squares indicate those above $18^{\prime}$. The dashed blue line, consisting of a horizontal segment at $1.1 \times 10^{-15} \mathrm{erg} \mathrm{s}^{-1} \mathrm{~cm}^{-2}$ and a linear increasing segment, approximates the sensitivity limit as a function of off-axis angle. The dotted blue box (the region above $6.8 \times 10^{-15} \mathrm{erg} \mathrm{s}^{-1} \mathrm{~cm}^{-2}$ and below 9'.6) contains the complete sample used in this study. 32 sources lie outside the plotted parameter space.

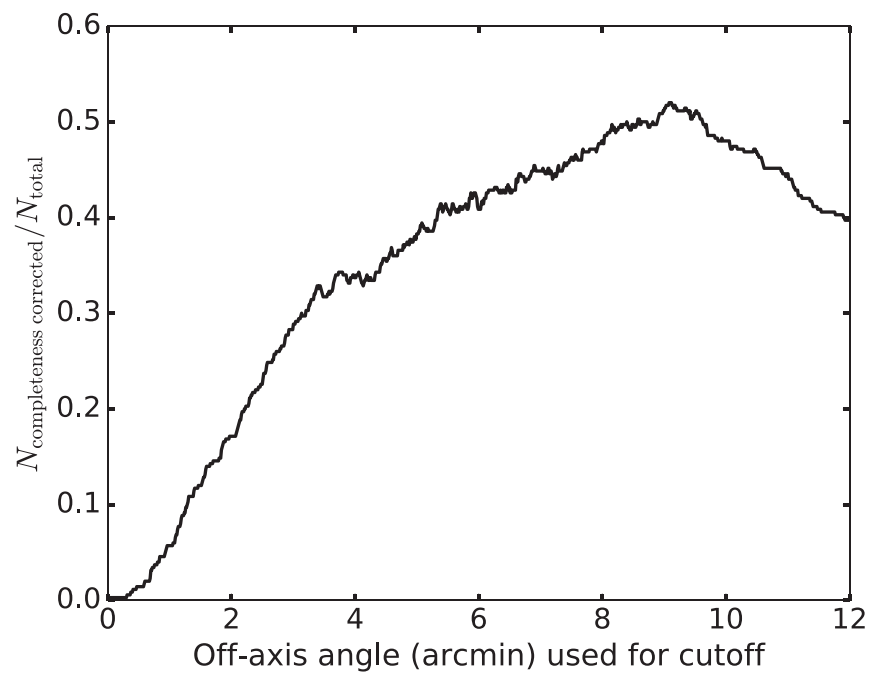

Figure 3. Fraction of $N_{\text {total }}=344$ sources that are included in the flux-limited complete sample for a chosen off-axis angle cutoff, using the dashed blue line in Figure 2.

variation in these distances allows for a larger coverage of galactocentric radii by a complete sample for a given off-axis angle cutoff. Due to the overlap between fields, six sources are 
detected in two observations. For these sources, we used the average flux and show the source at the smallest off-axis angle in Figure 2.

\subsection{Optical Data}

To distinguish field LMXBs from AGNs, LMXBs in GCs, and foreground stars, we used deep optical observations from the Next Generation Virgo Cluster Survey point source catalog (NGVS, Ferrarese et al. 2012) taken with MegaCam on the Canada-France-Hawaii Telescope. This survey detected 180692 optical sources with a depth of apparent magnitude $g \approx 25.9$ at $10 \sigma$ for point sources (Ferrarese et al. 2012).

At the distance of the Virgo Cluster, GCs and LMXBs can be treated as point sources, and the optical counterparts of AGNs are dominated by accretion light from the nucleus, and can also be treated as point sources. Therefore, for the purpose of our study, we used the completeness limit for point sources, rather than the shallower limit for large extended sources like galaxies in Virgo. The surface luminosity of NGC 4472 (Mihos et al. 2013) is fainter than the sky brightness outside a radius of a few arcminutes (and about 3 mag fainter in the outer parts), and is not a limiting factor. We searched the NGVS point source catalog out to a radial distance that matched the extent of the X-ray data.

For the inner region, we used the X-ray sources within the four Hubble Space Telescope fields described in Kundu et al. (2002).

\subsubsection{AGNs and GCs}

The vast majority of unobscured AGNs and unidentified X-ray sources in LaMassa et al. (2016) brighter than our X-ray completeness cutoff have $r \lesssim 25$. Almost all AGNs have $g-r<1$ (e.g., Richards et al. 2001), and the few above this typically have high redshifts. AGNs with redshifts larger than 3.5 have X-ray fluxes fainter than our cutoff limit in $97 \%$ of the Chandra Deep Field-South sample (Vito et al. 2013), which also used ACIS-I. This means that almost all AGNs have $g<26$, brighter than the NGVS detection limit for point sources. Systems dominated by galaxy starlight will have much higher ratios of optical to X-ray emission than unobscured AGNs. Based on the GC luminosity function of NGC 4472 and other elliptical galaxies, almost all GCs at the distance of NGC 4472 have $g<26$ (e.g., Rejkuba 2001; Kundu et al. 2002, 2007) and are expected to be listed in the NGVS catalog, and those that are fainter have low masses and are unlikely to host LMXBs. Therefore, both unobscured AGNs and LMXBs located in GCs are optically bright enough for the NGVS to see.

\subsubsection{Low-mass X-Ray Binaries}

Generally, LMXBs in GCs and AGNs have optical counterparts, whereas LMXBs in the field do not, but there are exceptions both ways. Here, we considered optically bright field LMXBs, and we took a conservative approach when rejecting contaminating sources (sources that could be confused with field LMXBs).

The four X-ray sources with full-band $(0.5-7 \mathrm{keV}) \mathrm{X}$-ray flux $f_{\mathrm{X}}>1 \times 10^{-13} \mathrm{erg} \mathrm{s}^{-1} \mathrm{~cm}^{-2}\left(L_{\mathrm{X}}>3 \times 10^{39} \mathrm{erg} \mathrm{s}^{-1}\right.$ if associated with NGC 4472) have clearly identified optical counterparts with $g<23$, and the optical to X-ray luminosity ratios are between 0.3 and 1.2. These four sources are most likely AGNs (one is confirmed as such by SIMBAD Astronomical Database) or possibly LMXBs in GCs.

All other X-ray sources have $f_{\mathrm{X}}=6.8 \times 10^{-15}-7.4 \times$ $10^{-14} \mathrm{erg} \mathrm{s}^{-1} \mathrm{~cm}^{-2}$. In this flux range, LMXBs with orbital periods below 10 days have $g>28$ according to the relation between optical and X-ray luminosity for a sample of NS and BH LMXBs by van Paradijs \& McClintock (1994). This is 1.5 mag fainter than the $50 \%$ completeness limit of the NGVS survey around NGC $4472\left(g_{\text {lim }}=26.5\right.$, S. Gwyn 2017, private communication). Therefore, virtually no LMXBs with orbital periods below 10 days should have been detected in the NGVS catalog. In the sample of LMXBs with known orbital periods in the catalog by Liu et al. (2007), seven out of 73 , about $10 \%$, have orbital periods longer than 10 days. ${ }^{8}$ In addition, LMXBs with $f_{\mathrm{X}}<2 \times 10^{-14} \mathrm{erg} \mathrm{s}^{-1} \mathrm{~cm}^{-2}$ and orbital periods $<30$ days also have $g>28$. Even at brighter magnitudes, $g=26-28$, many sources will be missing from the NGVS catalog given its $50 \%$ completeness limit of $g_{\lim }=26.5$.

Because about $10 \%$ of LMXBs in our sample have orbital periods longer than 10 days, enabling it to have $g<28$, and furthermore at most a few tens of percent of these is actually detected by NGVS given the incompleteness of the catalog at such faint luminosities, we expect only a few percent of field LMXBs in our sample to have a detected optical counterpart. This may result in some field LMXBs to be removed from the sample, but this error is smaller than several other errors in our analysis. Also, if the actual detection limit for the faintest sources is shallower than the conservative estimate of $g=28$, then the minimum orbital period LMXBs need to be optically bright enough to be detected becomes longer than 10 days, and even fewer field LMXBs will be removed.

The vast majority of LMXBs are expected to be too optically faint for the NGVS to see. They have no optical counterpart in the NGVS data, except for spurious matches. For the reasons described here and in Section 2.2.1, all X-ray sources with physically associated optical counterparts are assumed to be LMXBs in GCs or AGNs, and are removed from the sample. $\mathrm{X}$-ray sources without optical counterparts are assumed to be field LMXBs, which are our sources of interest. This way, the statistical number of field LMXBs in each annulus can be calculated, but because of spurious optical matches we do not attempt to identify individual X-ray sources.

\subsubsection{Matching X-Ray and Optical Sources}

We used the analysis tool TOPCAT (Taylor 2017) to crosscorrelate the X-ray and optical source catalogs using a $1^{\prime \prime}$ matching radius. A test showed this matching radius to be optimal. With a smaller matching radius, too many real counterparts are being missed, whereas using a larger radius gives almost no additional real counterparts, while errors become larger as a result of the strongly increasing number of chance matches. For our flux cutoff and corresponding photon count (about 16 net counts), even at large off-axis angles a $1^{\prime \prime}$ matching radius is sufficient to find almost all real counterparts. We also verified this by observing that the density of optical sources between $1^{\prime \prime}$ and $4^{\prime \prime}$ away from X-ray sources at large

\footnotetext{
8 In Liu et al. (2007), 60\% of LMXBs do not have a listed orbital period, but we found that the median $\mathrm{HI}$ extinction at their sky locations is 2.9 times higher than for LMXBs with known orbital periods. We think that extinction and crowding near the Galactic plane are the primary reasons why these systems do not have their orbital periods measured, rather than potential selection effects such as the difficulty in measuring very long and short orbital periods.
} 
off-axis angles is not significantly different from the density between $4^{\prime \prime}$ and $8^{\prime \prime}$. In Section 3, we included the effect of using different matching radii in the error estimates.

To estimate the rate of spurious associations, we also matched the X-ray and optical data sets after shifting by $5^{\prime \prime}$ in eight different directions (separated by $45^{\circ}$ angles). This displacement distance is large enough to assure that X-ray sources do not match with their real optical counterparts, and at the same time small enough that the variation in the space density of GCs changes negligibly over this angular displacement. Consequently, any remaining matches must be chance coincidences. The average number of spurious matches over these displacements is subtracted from the number of matches in the real data, and the resulting figure is taken to be the number of X-ray sources with physically associated optical counterparts.

\subsubsection{Optically Obscured AGNs}

AGNs can be too optically faint to be detected in optical data if they are obscured (e.g., Fabian 1999; Risaliti et al. 1999; Fiore et al. 2000; Moretti et al. 2003). Therefore, the remaining sample after optical elimination may still contain obscured AGNs.

We estimated the fraction of AGNs that are optically fainter than the NGVS limit using the Stripe 82 X-ray survey observations (LaMassa et al. 2016, VizieR Online Data Catalog). This survey targets blank fields, as it specifically avoids known targets such as nearby galaxies. It uses new and archival XMM-Newton and archival Chandra observations to observe $31.3 \mathrm{deg}^{2}$ within the Sloan Digital Sky Survey (SDSS) Stripe 82 Legacy field (Frieman et al. 2008), located within 1.5 of the celestial equator. The flux limit in the full band (0.5-7 keV for Chandra, 0.5-10 keV for XMM-Newton) is $\sim 2.1 \times 10^{-15} \mathrm{erg} \mathrm{s}^{-1} \mathrm{~cm}^{-2}$. There are $5972 \mathrm{X}$-ray sources brighter than our flux completeness cutoff. Of these, $25.8 \%$ do not have an optical counterpart in SDSS data, down to about 25-26th magnitude. As we only did optical matching, we did not consider the other multiwavelength data that are used by LaMassa et al. (2016). As the SDSS photometry is about 2 magnitudes shallower than the NGVS photometry, this percentage of obscured AGNs is an upper limit for our purposes ,as it would probably be lower with deeper photometry.

We also looked at the fraction of AGNs that are optically faint using two older and smaller studies that targeted other fields.

The Chandra Deep Survey (Hornschemeier et al. 2001) used ACIS-I to observe a field centered around the Hubble Deep FieldNorth field, and identified $82 \mathrm{X}$-ray sources with a full-band $(0.5-8 \mathrm{keV}) \mathrm{X}$-ray sensitivity of $\sim 3 \times 10^{-16} \mathrm{erg} \mathrm{s}^{-1} \mathrm{~cm}^{-2}$, and found counterparts to X-ray sources in $I$-band photometry down to twenty-sixth magnitude, and $R$-band to twenty-fifth magnitude. Nine X-ray sources have no optical or near-infrared counterpart, and the brightest of these is $8.2 \times 10^{-16} \mathrm{erg} \mathrm{s}^{-1} \mathrm{~cm}^{-2}$, almost an order of magnitude fainter than our completeness cutoff. The 10 sources brighter than our cutoff are all detected in both the $R$ - and $I$-bands, which are shallower than the NGVS photometry we used. $\mathrm{X}$-ray sources in the blank field are expected to be AGNs, and this result suggests that AGNs in our data at X-ray fluxes brighter than our completeness cutoff are successfully removed from the sample using NGVS optical counterparts, although the sample of 10 objects is small.

The Chandra Calán-Yale Deep Extragalactic Research (CYDER) survey (Treister et al. 2005) used deep ACIS-I and ACIS-S observations of five fields at different parts of the sky, and identified $267 \mathrm{X}$-ray sources, 119 of which have full-band (0.5-8 keV) X-ray sensitivity brighter than our completeness cutoff. Two ACIS-S fields were directed at galaxy groups, and we excluded the four sources brighter than our cutoff that were identified as galaxies in the study. Treister et al. (2005) looked for counterparts to X-ray sources in $V$-band photometry down to 25.5-26.7 magnitude $(5 \sigma)$, and $I$-band to 24.6-25.1 $(5 \sigma)$. These values may be slightly deeper than NGVS for some fields, depending on color and signal-to-noise conversions, but the CYDER X-ray exposure times are also longer. Of the remaining 115 sources brighter than our cutoff, 81 are detected in both bands, 15 in a single band (12 in $V$, three in $I$ ), and 19 are undetected. $19 / 115 \%=16.5 \%$ of $X$-ray sources have no optical/near-infrared counterpart, and again this fraction is an upper limit to the fraction using deeper optical data. Because some of the fields used are not blank but targeting compact groups of galaxies, it is also possible that some sources without an optical counterpart are LMXBs, or perhaps lensed AGNs in the background, implying a lower true fraction of obscured AGNs.

Based on these studies we find that a fraction $\sim 15 \%$ of AGNs in our data are expected to be obscured, and not be removed by optical matching.

\subsubsection{Sky Angular Density of AGNs}

We compared the number of AGNs behind NGC 4472 in our sample with AGN sky densities in other areas of the sky found by other studies. Due to large-scale cosmological structures, however, AGN density varies significantly across the sky (Vikhlinin \& Forman 1995; Cappi et al. 2001; Cappelluti et al. 2009). Therefore, these values are not a good way to determine the AGN density behind NGC 4472, which is more reliably done by the optical-counterpart method described in Section 2.2.1.

To estimate the AGN sky density, we used observations from the two surveys described in Section 2.2.4 that observed only blank fields. The Stripe $82 \mathrm{X}$ survey found 5972 X-ray sources brighter than our completeness cutoff, yielding a sky density of $\sim 300 \mathrm{deg}^{-2}$ based on LaMassa et al. (2016, Figure 3). (Following LaMassa et al. (2016), we multiplied our cutoff flux by 1.21 to convert the energy range from $0.5-7$ to $0.5-10 \mathrm{keV}$ ). The Chandra Deep Survey has 10 sources (not including sources identified as stars) brighter than our cutoff in a sky area of approximately $17^{\prime} \times 19^{\prime}$, yielding a density of $\sim 111 \mathrm{deg}^{-2}$. The CYDER (Treister et al. 2005) and ChaMP (Kim et al. 2007) surveys have higher sky densities $\left(300-400 \mathrm{deg}^{-2}\right)$ than we found using the same completeness cutoff. In addition to cosmic variation, this can be due to these surveys making use of fields likely to contain foreground sources associated with the actual targets of the observations. Different models in converting count rate to X-ray flux can also play a role in variation in sky density.

Sky densities of important classes of sources are plotted against galactocentric radius in Figure 4. The semimajor axis distance of our Chandra observations extends to almost $30^{\prime}$, but the galactocentric radii of sources do not extend beyond $25^{\prime}$. This is in part due to the distribution of LMXBs with 


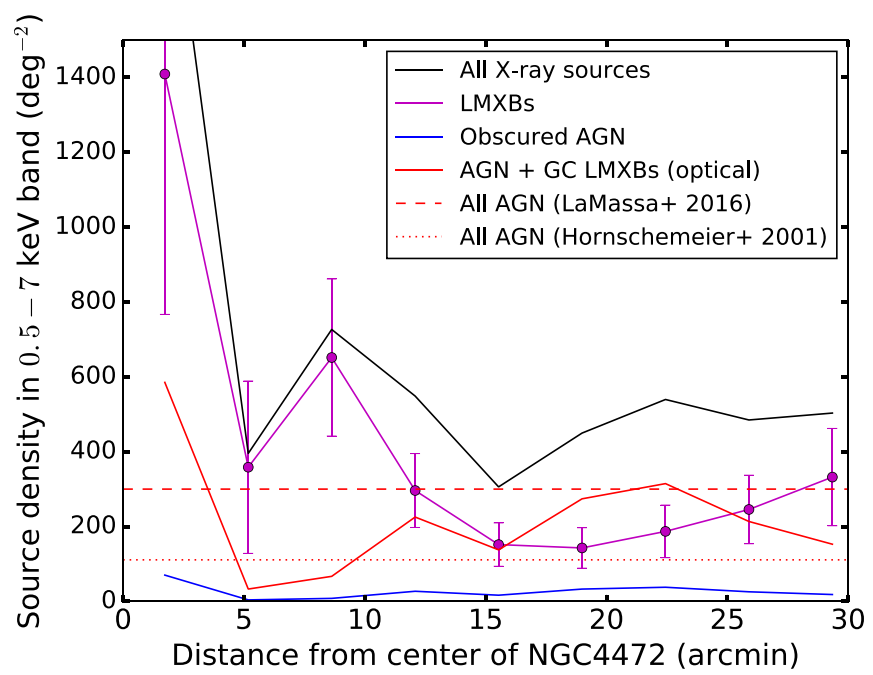

Figure 4. Sky angular density of X-ray sources as a function of galactocentric radius. The projected distance is the semimajor axis of the elliptical annuli. The total density of X-ray sources (black curve) is the sum of field LMXBs (magenta curve), obscured AGNs (blue curve), and optically detected AGNs plus LMXBs in GCs (i.e., the X-ray sources with optical counterpart indicated by the solid red curve). The background AGN sky densities brighter than our completeness cutoff flux of $6.8 \times 10^{-15} \mathrm{erg} \mathrm{s}^{-1} \mathrm{~cm}^{-2}$, based on two studies of blank fields in different parts of the sky (not the Virgo Cluster), are shown as horizontal dashed and dotted red lines. The LaMassa et al. (2016) figure is an upper limit, as it includes all X-ray sources in the sample. Error bars on the data points indicate $1 \sigma$ confidence intervals. For clarity, these are only shown on the field LMXB distribution.

azimuthal angle peaking near the east-west axis, about $55^{\circ}$ away from the major axis of the light distribution. Depending on location, the density of AGNs plus LMXBs in GCs is somewhat lower or similar to the density of AGNs from LaMassa et al. (2016), which is by far the largest study of the two indicated by horizontal red lines. Overall the AGN densities are consistent with cosmic variation, especially as our field of view is only $0.4 \mathrm{deg}^{2}$. The density of AGNs plus LMXBs in GCs in our sample at galactocentric radii of $4^{\prime}-10^{\prime}$ is lower than the LaMassa et al. (2016) value. This is partly due to the small number statistics, because this part of the galaxy is covered only by the outer parts of the halo CCDs, which are aimed at $\sim 15^{\prime}$ from the Galactic center. Other complicating factors in the inner part of the galaxy are X-ray emission from diffuse gas, obscuration by dust, and crowding of X-ray and optical sources. Within $3^{\prime}$ from the center of the galaxy, the high sky density of GCs will contribute significantly to the number of X-ray sources with optical match.

Because X-ray sources associated with GCs also have optical counterparts, it is not known precisely how many X-ray sources with optical counterparts are GCs, and how many are unobscured AGNs. The number of obscured AGNs follows from the (unknown) number of unobscured AGNs combined with the fraction of AGNs that are obscured (Section 2.2.4), and cannot be calculated exactly. Because at most galactocentric radii, the density of AGNs by LaMassa et al. (2016) is comparable to the unobscured AGNs plus GC LMXB density in our result, it is probable that most of the sources with optical counterparts are AGNs rather than GCs. We conservatively assumed this to be $80 \%$ in our calculations (see Section 4 for a justification).
The number density of obscured AGNs that we estimated this way, assuming that $15 \%$ of AGNs are obscured even at the depth of the NGVS optical data (based on the surveys in Section 2.2.4), is shown as the blue curve in Figure 4. There is a considerable uncertainty in the fraction of AGNs that are obscured as a result of differences in the depths of optical and $\mathrm{X}$-ray observations between studies. This uncertainty is taken into account in the error estimate of the results. The obscured AGN density (blue curve) lies significantly below the LMXB density (magenta curve). This implies that obscured AGNs are not likely to cause a significant contamination of the field LMXB density estimate.

\subsection{Surface Brightness}

The surface brightness of NGC 4472 decreases significantly from the center to the outer halo (Mihos et al. 2013, Figure 2, left panel). We considered the number of field LMXBs per unit stellar light, which approximates stellar mass. We calculated this ratio by dividing the total number of field LMXBs in the annulus by the luminosity of diffuse starlight within the annulus, derived from the surface brightness profile given in Mihos et al. (2013).

\section{Results}

We identified 344 point-like X-ray sources in NGC 4472, including 302 in the halo in the five new Chandra observations, as well as an additional 42 sources in archival data from the inner part of the galaxy. Our flux-limited complete sample consists of $174 \mathrm{X}$-ray sources across all six observations, 86 with an optical counterpart within $1^{\prime \prime}$ (either physically associated or chance superpositions), and 88 without an optical counterpart. Among the 174 sources we estimated $91 \pm 15$ LMXBs, $74 \pm 14$ X-ray sources with a physically associated optical counterpart (LMXBs in GCs and AGNs), and $9 \pm 5$ obscured AGNs. $12 \pm 5$ X-ray sources with optical counterparts are false superpositions. All errors indicate $1 \sigma$ confidence intervals. The errors in Figure 4 are larger due to larger Poisson errors for the individual annuli compared to the entire sample (Kraft et al. 1991). The X-ray sources in our flux-limited sample have a lower limit of $6.8 \times 10^{-15} \mathrm{erg} \mathrm{s}^{-1} \mathrm{~cm}^{-2}$ for the $0.5-7 \mathrm{keV}$ band, or $2 \times 10^{38} \mathrm{erg} \mathrm{s}^{-1}$ for sources located in NGC 4472, and therefore are more luminous than the Eddington limit for a $1.4 M_{\odot}$ NS accreting hydrogen-rich matter.

Figure 5 shows the number of field LMXBs per unit $V$-band luminosity (Section 2.3) for each annulus. The uncertainties in these field LMXB densities are a combination of Poisson errors regarding the number of sources per annulus, of the dependency of the result on the optical matching radius (Section 2.2.3), and of the fraction of AGNs that are obscured (Section 2.2.4).

Figure 5 also shows the $B-V$ color as a function of galactocentric radius (Mihos et al. 2013). These colors indicate that metallicity decreases with galactocentric radius, and that the outer halo is very metal-poor. A color of $B-V=0.7$ corresponds to $[\mathrm{Fe} / \mathrm{H}]<-1$ (Mihos et al. 2013).

There is an anticorrelation between color and the number of field LMXBs per unit stellar light. This implies an anticorrelation between number of field LMXBs and metallicity, in the sense that there are more field LMXBs per unit stellar light 


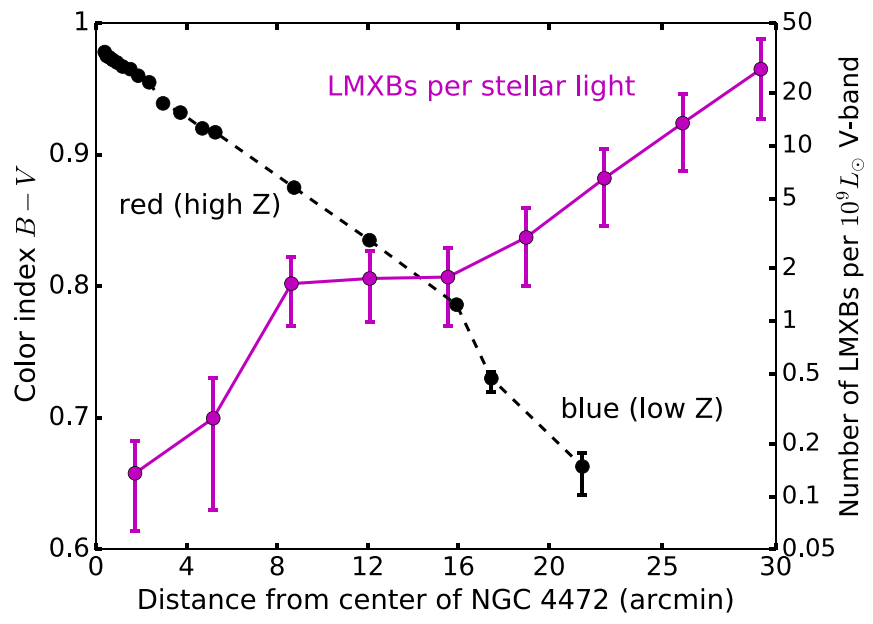

Figure 5. Radial distribution of field LMXBs with $L_{X}>2 \times 10^{38} \mathrm{erg} \mathrm{s}^{-1}$ per unit stellar light (magenta circles connected by solid curve, axis on the right), after eliminating LMXBs in GCs and AGNs. The projected distance is the semimajor axis of the elliptical annuli. This corresponds to the solid magenta curve in Figure 4. The color index (black circles connected by dashed curve, axis on the left) and surface brightness data are taken from Mihos et al. (2013). Error bars on the data points indicate $1 \sigma$ confidence intervals.

at bluer colors. This is the opposite of the metallicity effect seen in GCs.

A $\chi^{2}$ test comparing the number of field LMXBs per unit stellar light to a constant distribution equal to its weighted average (with weight factors of error $^{-2}$ ) yields $\chi^{2} /$ dof $=29.9 / 8=3.7$ for nine bins, corresponding to a $p$ value of $2 \times 10^{-4}$. The probability that the field LMXB density per unit stellar light is consistent with a constant value based on a Kolmogorov-Smirnov test is $p=2.0 \times 10^{-5}$. This test yields a statistic $D=0.626$ for the cumulative distribution of $V$-band light in each annulus versus the distribution of field LMXBs in each annulus, when taking 50 bins for the field LMXB distribution (instead of nine) to obtain a more significant result, and 19 bins for the flux distribution (Mihos et al. 2013, Figure 2, left panel). Both tests indicate that the number of field LMXBs per unit stellar light is significantly increasing with galactocentric radius.

Other choices for the off-axis angle cutoff lead to different complete samples. In the case of a smaller cutoff angle, fainter sources are included, but sources that are farthest off-axis are excluded (Figure 2). This improves the quality of the sample at galactocentric radii that are relatively close to those of the aimpoints of the halo observations, but comes at the cost of losing information at large galactocentric radii. When the offaxis angle cutoff differs from $9 ! 6$, the number of sources retained in the sample is lower (per Figure 3). Figure 6 shows the same radial distribution of field LMXBs as in Figure 5 (magenta curve), but now compares it with two more fluxlimited samples; one with a lower off-axis angle cutoff and a correspondingly lower flux limit (dashed-dotted green), and a sample with a higher off-axis angle cutoff and a higher flux limit (dashed blue). Table 2 lists the off-axis angles and flux limits for all three samples, as well as the statistics for the anticorrelation between color and field LMXBs per unit light. The main results are similar; the number of field LMXBs per unit light still increases significantly with galactocentric radius. The statistical tests for a lower off-axis cutoff of 5!4 are less significant than those for our standard sample with an off-axis cutoff of 9!6. This is partly due to a lack of coverage of

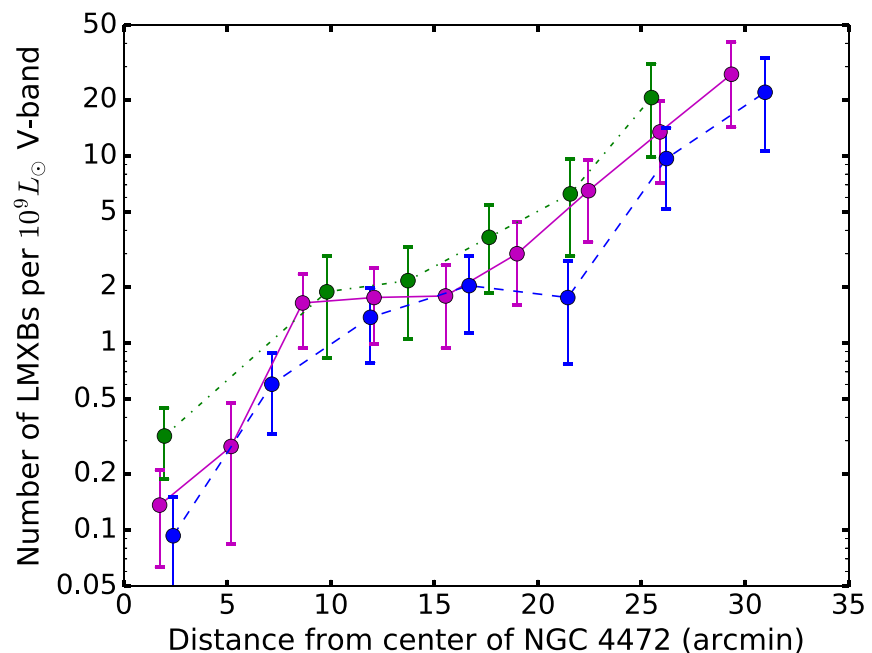

Figure 6. Radial distribution of field LMXBs per unit stellar light after eliminating LMXBs in GCs and AGNs. The solid magenta curve is identical to the one in Figure 5. The dashed-dotted green and dashed blue curves represent samples with lower (5!4) and higher $\left(12^{\prime}\right)$ off-axis angle cutoffs, listed in Table 2. The projected distance is the semimajor axis of the elliptical annuli. Error bars on the data points indicate $1 \sigma$ confidence intervals.

Table 2

Parameters for Three Samples of LMXBs, and Statistical Test Results for the Anticorrelation between Color and Number of Field LMXBs Per Unit Stellar Light

\begin{tabular}{lccccc}
\hline \hline OAA & $F$ & $\chi^{2} /$ dof & $p\left(\chi^{2}\right)$ & KS & $p(\mathrm{KS})$ \\
\hline $5 ! 4$ & 3.0 & $16.2 / 5=3.2$ & $6 \times 10^{-3}$ & 0.525 & $7 \times 10^{-4}$ \\
$9 ! 6$ & 6.8 & $29.9 / 8=3.7$ & $2 \times 10^{-4}$ & 0.626 & $2 \times 10^{-5}$ \\
$12^{\prime}$ & 9.0 & $23.3 / 6=3.4$ & $7 \times 10^{-4}$ & 0.633 & $2 \times 10^{-5}$ \\
\hline
\end{tabular}

Note. OAA stands for off-axis angle, $F$ is the lower flux limit in units of $10^{-15} \mathrm{erg} \mathrm{s}^{-1} \mathrm{~cm}^{-2}$. See the text for more details.

galactocentric radii in the range of $4^{\prime}-8^{\prime}$ and partly due to a smaller overall sample size.

The near-infrared $K$-band is a better indicator for mass than optical light (e.g., Bell \& de Jong 2001). Using the relation between $B-V$ and $V-K$ in Pecaut \& Mamajek (2013, Table 5), it shows that $V-K$ decreases from the inner to the outer parts of the galaxy. Therefore, the surface brightness in the $K$-band falls off steeper with radius than the $V$-band surface brightness. This means that the anticorrelation result will become more significant when expressed in units of $K$-band light rather than $V$-band light, as we did in Figures 5 and 6.

The number of field LMXBs $N$ per unit stellar light for our standard sample with off-axis angle cutoff $9.6^{\prime}$ can be fitted by $\log _{10}\left(N / L_{V \odot}\right)=(0.076 \pm 0.007) r-(10.01 \pm 0.13), \quad$ with $L_{V \odot}$ the solar $V$-band luminosity and $r$ the galactocentric radius in arcmin. The relation between $N$ and color is fitted by $\log _{10}\left(N / L_{V \odot}\right)=(-4.7 \pm 0.5)(B-V)-(5.3 \pm 0.4) . \quad$ The uncertainties in these fits indicate $1 \sigma$ statistical errors. The $1 \sigma$ relative error on the slope in the $\log _{10}\left(N / L_{V \odot}\right)$ versus $r$ relation of $9 \%$ is another indication that this slope being positive is significant. The slope of the $\log _{10}\left(N / L_{V \odot}\right)$ versus $B-V$ relation being negative is also significant.

\section{Discussion}

Our assumptions in Section 2.2.5 that $80 \%$ of sources with an optical counterpart are AGNs, and $15 \%$ of AGNs are 
obscured are probably conservative. Lowering either number decreases the estimated number of obscured AGNs (blue curve in Figure 4) and increases the estimated number of field LMXBs (magenta curve), and makes the result that the number of field LMXBs per unit stellar light increases with galactocentric radius statistically more significant. Assuming the most conservative percentage of $100 \%$ instead of $80 \%$ (i.e., assuming there are no GC X-ray sources) produces a result that is almost equally significant, because the corresponding increase in the absolute number of obscured AGNs (15\% of all AGNs) is small. Even in the extreme case in which $100 \%$ of optical sources are AGNs, and $75 \%$ of AGNs are obscured instead of $15 \%$ (which is certainly not realistic for our X-ray flux limit combined with the deep optical NGVS observations, according to the AGN studies cited in Section 2.2.4) the KS test still gives $1.0 \times 10^{-3}$ instead of $2.0 \times 10^{-5}$.

If LMXBs are predominantly formed at the galactocentric radii where they are found today, their high number density per unit light in the metal-poor outer halo would require a reversed metallicity effect, opposite from that seen in GCs. Mihos et al. (2013) considered it very unlikely that the steep color gradient in NGC 4472 is a consequence of a decrease in stellar ages (rather than metallicity) with galactocentric radius because it would require a very large and relatively recent merger event, which they found to be inconsistent with the observed accretion features in the galaxy. Merger events of any magnitude can initiate local star formation and potentially lead to an excess of LMXBs at larger galactocentric radii, but we did not find clear evidence for an azimuthal pattern in the LMXB distribution. Natal kicks may explain the observed field LMXB distribution, if most binaries formed in the inner galaxy where most of the stellar mass is, and many of the surviving systems reached the outer halo after receiving kicks. However, the required kicks may be larger than those inferred from Galactic radio pulsars (Hobbs et al. 2005). Many LMXBs, even those from the higher (non-electron capture) peak in the kick velocity distribution (e.g., Arzoumanian et al. 2002), could have been retained in the galaxy due to the deep potential of NGC 4472, making this massive galaxy a good test location for natal kicks on NSs and BHs. Because the lower limit on the flux of $6.8 \times$ $10^{-15} \mathrm{erg} \mathrm{s}^{-1} \mathrm{~cm}^{-2}$ in our standard sample corresponds to the Eddington limit for NSs, many of the LMXBs in the complete sample may host a $\mathrm{BH}$ accretor.

Another possibility is that some field LMXBs originate from GCs from which they were dynamically ejected, or which were evaporated (Kundu et al. 2002, 2007). However, Juett (2005) and Irwin (2005) found that a significant number of LMXBs in elliptical galaxies must have formed in the field rather than in GCs, and Kim et al. (2006) did not find evidence either for or against the idea that all LMXBs are formed in GCs. Also, kick velocities complicate comparison of radial profiles of GCs and field LMXBs, which in addition are usually not available for the outer halo, which we studied.

\subsection{Previous Studies of Extragalactic Halos}

Several studies also found an increase in the number of LMXBs per unit stellar light toward larger galactocentric radii, although these did not extend as far out as our data.

In the halo of M104, Li et al. (2010) found an excess of $\mathrm{X}$-ray sources compared to starlight between galactocentric radii of $4^{\prime}-9^{\prime}(10-24 \mathrm{kpc}$, based on a distance of $9.0 \mathrm{Mpc}$, Spitler et al. 2006), double the number expected from background AGNs. As possible explanations, the authors considered ejection from inner region of the galaxy as a result of supernova kicks, association with GCs, formation following a recent galaxy merger, or a strong overdensity in the AGN background. Li et al. (2010) subsequently also found excesses in some other galaxies.

Zhang et al. (2013) found an excess compared with stellar light in the outskirts of 20 galaxies, at radii of $\sim 2^{\prime}-9^{\prime}$, and concluded that both galaxy mass and LMXBs in GCs contribute. In NGC 4365, the authors were able to eliminate LMXBs in GCs using optical counterparts, and found an excess of field LMXBs between 2!5-6!4 (15-38 kpc).

Mineo et al. (2014) also found an excess of LMXBs (that could be either field or GC sources) compared with $V$-band stellar light up to $7.5(\sim 37 \mathrm{kpc})$ from the center in the outskirts of NGC 4649 (M60), which has a distance of 16.8 Mpc (Tonry et al. 2001). They suggested galaxy interaction causing a rejuvenated field LMXB population as explanation, but LMXBs in GCs may also play a role due to lack of deep GC data at large radii. Mineo et al. (2014) considered natal kicks to be unlikely as main cause due to the shape of their source distribution, which spikes near $6.5-7^{\prime}$ from the center of NGC 4649.

\section{Summary}

We obtained Chandra X-ray observations of the outer halo of NGC 4472, and combined with existing X-ray and groundbased optical data, we showed that the number of field LMXBs per unit stellar light increases significantly from the nucleus toward the outer halo. Migration of binaries caused by natal kicks onto NSs and BHs can possibly explain this in part, but the variation in metallicity across the halo may also play a role.

The authors thank Stephanie LaMassa for providing data from the Stripe 82X survey; Stephen Gwyn and the Next Generation Virgo Cluster Survey team for providing optical data; and Ezequiel Treister, Jay Strader, Gregory Sivakoff, and Vlad Tudor for useful discussion. Support for this work was provided by NASA through Chandra Award Numbers GO415034 and GO5-16084 issued by the Chandra X-ray Center (CXC), which is operated by the Smithsonian Astrophysical Observatory for and on behalf of NASA under contract NAS803060. The scientific results reported in this article are based on observations made by the Chandra X-ray Observatory, and on data obtained from the Chandra Data Archive. This research has made use of software provided by the CXC in the application package CIAO, as well as NASA's Astrophysics Data System Bibliographic Services.

Facility: CXO.

Software: CIAO (Fruscione et al. 2006), TOPCAT (Taylor 2017).

\section{ORCID iDs}

Lennart M. van Haaften (iD https://orcid.org/0000-00015422-6775

J. Christopher Mihos (iD https://orcid.org/0000-0002-7089-8616

David J. Sand (10 https://orcid.org/0000-0003-4102-380X

\section{References}

Angelini, L., Loewenstein, M., \& Mushotzky, R. F. 2001, ApJL, 557, L35 Arzoumanian, Z., Chernoff, D. F., \& Cordes, J. M. 2002, ApJ, 568, 289 
Baes, M., Sil'chenko, O. K., Moiseev, A. V., \& Manakova, E. A. 2007, A\&A, 467, 991

Bell, E. F., \& de Jong, R. S. 2001, ApJ, 550, 212

Bellazzini, M., Pasquali, A., Federici, L., Ferraro, F. R., \& Pecci, F. F. 1995, ApJ, 439, 687

Brandt, W. N., Podsiadlowski, P., \& Sigurdsson, S. 1995, MNRAS, 277, L35

Cappelluti, N., Brusa, M., Hasinger, G., et al. 2009, A\&A, 497, 635

Cappi, M., Mazzotta, P., Elvis, M., et al. 2001, ApJ, 548, 624

Clark, G. W. 1975, ApJL, 199, L143

Dickey, J. M., \& Lockman, F. J. 1990, ARA\&A, 28, 215

Fabian, A. C. 1999, MNRAS, 308, L39

Ferrarese, L., Côté, P., Cuillandre, J.-C., et al. 2012, ApJS, 200, 4

Fiore, F., La Franca, F., Vignali, C., et al. 2000, NewA, 5, 143

Fragos, T., Kalogera, V., Willems, B., et al. 2009, ApJL, 702, L143

Freeman, P. E., Kashyap, V., Rosner, R., \& Lamb, D. Q. 2002, ApJS, 138, 185

Frieman, J. A., Bassett, B., Becker, A., et al. 2008, AJ, 135, 338

Fruscione, A., McDowell, J. C., Allen, G. E., et al. 2006, SPIE Proc., 6270, $62701 \mathrm{~V}$

Gilfanov, M. 2004, MNRAS, 349, 146

Grindlay, J. E. 1987, in IAU Symp. 125, The Origin and Evolution of Neutron Stars, ed. D. J. Helfand \& J.-H. Huang (Dordrecht: Reidel), 173

Grindlay, J. E. 1993, in ASP Conf. Ser. 48, The Globular Cluster-Galaxy Connection, ed. G. H. Smith \& J. P. Brodie (San Francisco, CA: ASP), 156

Hjellming, R. M., \& Rupen, M. P. 1995, Natur, 375, 464

Hobbs, G., Lorimer, D. R., Lyne, A. G., \& Kramer, M. 2005, MNRAS, 360, 974

Hornschemeier, A. E., Brandt, W. N., Garmire, G. P., et al. 2001, ApJ, 554,742

Iben, I., Jr., Tutukov, A. V., \& Fedorova, A. V. 1997, ApJ, 486, 955

Ingram, A., Done, C., \& Fragile, P. C. 2009, MNRAS, 397, L101

Irwin, J. A. 2005, ApJ, 631, 511

Ivanova, N. 2006, ApJ, 636, 979

Ivanova, N., Fragos, T., Kim, D.-W., et al. 2012, ApJL, 760, L24

Janowiecki, S., Mihos, J. C., Harding, P., et al. 2010, ApJ, 715, 972

Juett, A. M. 2005, ApJL, 621, L25

Kalberla, P. M. W., Burton, W. B., Hartmann, D., et al. 2005, A\&A, 440, 775

Katz, J. I. 1975, Natur, 253, 698

Kim, E., Kim, D.-W., Fabbiano, G., et al. 2006, ApJ, 647, 276

Kim, M., Wilkes, B. J., Kim, D.-W., et al. 2007, ApJ, 659, 29

Kormendy, J., Fisher, D. B., Cornell, M. E., \& Bender, R. 2009, ApJS, 182,216

Kraft, R. P., Burrows, D. N., \& Nousek, J. A. 1991, ApJ, 374, 344
Kundu, A., Maccarone, T. J., \& Zepf, S. E. 2002, ApJL, 574, L5

Kundu, A., Maccarone, T. J., \& Zepf, S. E. 2007, ApJ, 662, 525

LaMassa, S. M., Urry, C. M., Cappelluti, N., et al. 2016, ApJ, 817, 172

Li, Z., Spitler, L. R., Jones, C., et al. 2010, ApJ, 721, 1368

Liu, Q. Z., van Paradijs, J., \& van den Heuvel, E. P. J. 2007, A\&A, 469, 807

Lyne, A. G., \& Lorimer, D. R. 1994, Natur, 369, 127

Maccarone, T. J., Kundu, A., \& Zepf, S. E. 2004, ApJ, 606, 430

Mihos, J. C., Harding, P., Rudick, C. S., \& Feldmeier, J. J. 2013, ApJL, 764, L20

Miller-Jones, J. C. A., Jonker, P. G., Nelemans, G., et al. 2009, MNRAS, 394, 1440

Mineo, S., Fabbiano, G., D’Abrusco, R., et al. 2014, ApJ, 780, 132

Moretti, A., Campana, S., Lazzati, D., \& Tagliaferri, G. 2003, ApJ, 588, 696

Pecaut, M. J., \& Mamajek, E. E. 2013, ApJS, 208, 9

Pfahl, E., Rappaport, S., Podsiadlowski, P., \& Spruit, H. 2002, ApJ, 574,364

Podsiadlowski, P., Langer, N., Poelarends, A. J. T., et al. 2004, ApJ, 612, 1044

Reid, M. J., McClintock, J. E., Steiner, J. F., et al. 2014, ApJ, 796, 2

Rejkuba, M. 2001, A\&A, 369, 812

Repetto, S., \& Nelemans, G. 2015, MNRAS, 453, 3341

Richards, G. T., Fan, X., Schneider, D. P., et al. 2001, AJ, 121, 2308

Risaliti, G., Maiolino, R., \& Salvati, M. 1999, ApJ, 522, 157

Sarazin, C. L., Irwin, J. A., \& Bregman, J. N. 2000, ApJL, 544, L101

Sarazin, C. L., Kundu, A., Irwin, J. A., et al. 2003, ApJ, 595, 743

Spitler, L. R., Larsen, S. S., Strader, J., et al. 2006, AJ, 132, 1593

Tauris, T. M., \& van den Heuvel, E. P. J. 2006, in Formation and Evolution of Compact Stellar X-ray Sources, ed. W. H. G. Lewin \& M. van der Klis (Cambridge: Cambridge Univ. Press), 623

Taylor, M. 2017, Informatics, 4, 18

Thomas, D., Maraston, C., Bender, R., \& Mendes de Oliveira, C. 2005, ApJ, 621,673

Tonry, J. L., Dressler, A., Blakeslee, J. P., et al. 2001, ApJ, 546, 681

Treister, E., Castander, F. J., Maccarone, T. J., et al. 2005, ApJ, 621, 104

Trinchieri, G., \& Fabbiano, G. 1985, ApJ, 296, 447

van den Heuvel, E. P. J. 2007, in AIP Conf. Ser. 924, The Multicolored Landscape of Compact Objects and their Explosive Origins, ed. T. di Salvo et al. (Melville, NY: AIP), 598

van Paradijs, J., \& McClintock, J. E. 1994, A\&A, 290, 133

Vikhlinin, A., \& Forman, W. 1995, ApJL, 455, L109

Vito, F., Vignali, C., Gilli, R., et al. 2013, MNRAS, 428, 354

Zhang, Z., Gilfanov, M., \& Bogdán, Á 2013, A\&A, 556, A9 